\title{
Estrutura populacional da raça Girolando
}

\author{
Population structure of Girolando breed
}

\author{
Ali William Canaza-Cayo ${ }^{\mathrm{I}}$ Paulo Sávio Lopes $^{\mathrm{I}^{*}}$ \\ Marcos Vinicius Gualberto Barbosa da SilvaII Jaime Araújo CobuciII \\ Robledo de Almeida Torres ${ }^{I}$ Marta Fonseca Martins ${ }^{\text {II }}$ Wagner Antonio Arbex ${ }^{\text {II }}$
}

\section{RESUMO}

\begin{abstract}
O objetivo neste estudo foi avaliar a estrutura genética da população de bovinos da raça Girolando no Brasil. Analisou-se o arquivo de pedigree de 26.969 animais, composto de 3.031 machos e 23.938 fêmeas. $O$ nível de conteúdo de informação do pedigree na geração atual foi $61 \%$, mostrando ser de qualidade moderada. O coeficiente de endogamia médio e o coeficiente de relação médio da população Girolando foram 0,11 e $0,13 \%$, respectivamente. O tamanho efetivo da população, considerando a geração completa traçada, foi 188, acima do nível crítico. Do total de 9.457 ancestrais que contribuíram para a população de referência, 457 explicaram 50\% da variabilidade genética da população. O número efetivo de fundadores foi 551 e o de ancestrais 393. O intervalo médio de geração foi de 5,26 anos, sendo ligeiramente maior nas trilhas gaméticas mãe-filho e pai-filha. A partir dos coeficientes estimados, pode-se concluir que a endogamia nos rebanhos da raça Girolando foi de pequena magnitude e que as práticas de acasalamento foram adequadas durante o período avaliado. No entanto, é importante continuar com o monitoramento desses coeficientes a fim de prevenir perda de variabilidade genética.
\end{abstract}

Palavras- chave: coeficiente de endogamia, Girolando, tamanho efetivo populacional.

\section{ABSTRACT}

The aim of this study was to evaluate the population structure of Girolando cattle in Brazil. The pedigree file contained 26,969 individuals, from which 3,031 were males and 23,938 were females. The average level of completeness of the pedigree in the current generation was of reasonable quality (61\%). Inbreeding and average relatedness coefficients were low: 0.11 and $0.13 \%$, respectively. Estimates of effective population size considering the full generations traced was 188, which is above the critical level range. The number of ancestors that contributed to the reference population was 9,457 animals, from which 457 explained $50 \%$ of the genetic variability of the population. The effective number of founders and the effective number of ancestors in this population were, respectively, 551 and 393. The average generation interval was 5.26 years, slightly higher in genetic pathways dam-son and sire-daughter. The inbreeding in the Girolando breed was of small magnitude, indicating that the current practices of mating were adequate during the study period. However, it is important to continue monitoring these coefficients in order to prevent loss of genetic variability.

Key words: inbreeding, effective population size, Girolando.

\section{INTRODUÇÃO}

Por volta das décadas de 1940 e 1950, produtores de gado de leite começaram os cruzamentos entre animais das raças Gir e Holandesa com o intuito de obter animais que aliassem a alta capacidade de produção de leite do gado Holandês e a rusticidade da raça Gir.

Em 1989, o Ministério da Agricultura, Pecuária e Abastecimento (MAPA), juntamente com as Associações representativas, traçou as normas para formação do Girolando - Gado Leiteiro Tropical (5/8 Holandês + 3/8 Gir), transformando-o em prioridade nos programas de melhoramento genético de gado leiteiro. Em 1996, com a oficialização da raça Girolando pelo MAPA, a Associação Brasileira dos Criadores de Girolando (ABCG) foi encarregada de executar, desenvolver e acompanhar

\footnotetext{
'Departamento de Zootecnia, Universidade Federal de Viçosa (UFV), 36570-000, Viçosa, MG, Brasil. E-mail: plopes@ufv.br. *Autor para correspondência.

${ }^{\text {IE} E m b r a p a ~ G a d o ~ d e ~ L e i t e, ~ J u i z ~ d e ~ F o r a, ~ M G, ~ B r a s i l . ~}$

IIIDepartamento de Zootecnia, Universidade Federal do Rio Grande do Sul (UFRGS), Porto Alegre, RS, Brasil. 
o Registro Genealógico e as Provas Zootécnicas da raça, passando, então, a coletar e avaliar dados de características de produção, reprodução, conformação e manejo (SILVA et al., 2010).

O Brasil produz aproximadamente 25 bilhões de litros de leite fluido, sendo o $6^{\circ}$ produtor mundial, com o terceiro maior rebanho de vacas leiteiras do mundo, composto de cerca de 15 milhões de cabeças, em sua maioria da raça Girolando, com seus diversos grupos genéticos ou puro-sintéticos $5 / 8$, responsáveis por mais de $80 \%$ do leite produzido no Brasil (ABCG, 2011).

Durante os últimos anos, a intensidade de seleção, principal fator contribuinte do aumento de endogamia, tem sido intensificada na raça Girolando, que, aliada ao uso de novas tecnologias reprodutivas (inseminação artificial, transferência de embriões e fecundação in vitro), tem resultado em menor proporção de animais selecionados para serem progenitores da próxima geração. Isso tem acelerado o progresso genético na raça, mas também pode estar reduzindo a diversidade genética pelo aumento da endogamia.

O nível de endogamia, assim como seu efeito em características de produção e de reprodução em bovinos leiteiros, tem sido objeto de estudos de muitos pesquisadores (CORRALES et al., 2011; EAGLEN et al., 2012; SANTANA Jr. et al., 2012). Durante os últimos anos, têm sido publicados artigos sobre estudos de endogamia em bovinos de leite (REIS FILHO et al., 2010). No entanto, ainda não foram publicados estudos de endogamia na raça Girolando.

Este estudo foi realizado com os objetivos de avaliar a estrutura genética da população, particularmente os níveis e as tendências de endogamia, na raça Girolando no Brasil.

\section{MATERIAL E MÉTODOS}

Os dados de pedigree utilizados foram provenientes do Arquivo Zootécnico Nacional de Gado de Leite e fornecidos pelo Programa de Melhoramento Genético da Raça Girolando (PMGG), sob coordenação da Embrapa Gado de Leite, em parceria com a Associação Brasileira dos Criadores de Girolando (ABCG). O arquivo de pedigree incluiu 26.969 animais da raça Girolando (23.938 fêmeas e 3.031 machos) nascidos entre 1979 e 2008. Desses animais, 10.586 possuíam um ou ambos os pais desconhecidos e 16.383 formaram a população de referência, com ambos os pais conhecidos. O número de animais sem data de nascimento foi 14.530 e maior no período de 1979 a 1987.

As informações de pedigree na população Girolando foram avaliadas considerando os seguintes indicadores: i) o número de ancestrais conhecidos por geração e o índice de conteúdo de informação do pedigree, proposto por MACCLUER et al. (1983) como medida global da integridade do pedigree, ii) o número de gerações completas traçadas, iii) o número máximo de gerações traçadas; e iv) o número equivalente de gerações completas.

Neste estudo, foram consideradas 11 gerações de ancestrais e a descrição da abrangência de cada ancestral no pedigree foi feita até a quinta geração.

O número de gerações completas traçadas refere-se à geração mais distante em que todos os ancestrais sejam conhecidos, ou seja, que separa a progênie da mais distante geração. O número máximo de gerações traçadas é o número de gerações que separam o indivíduo de seu ancestral mais remoto. $\mathrm{O}$ número equivalente de gerações completas, por sua vez, é obtido pelo somatório dos termos $(1 / 2)^{\mathrm{n}}$ de todos os ancestrais conhecidos, em que n é o número de gerações que separa o indivíduo de cada ancestral conhecido (GUTIÉRREZ \& GOYACHE, 2005).

$O$ coeficiente de endogamia $(F)$ foi estimado de acordo com a metodologia de MEUWISSEN \& LUO (1992), que descreve a probabilidade de que um indivíduo carregue dois alelos idênticos por descendência. $\mathrm{O}$ incremento de endogamia (DF), calculado para cada geração, foi obtido utilizando-se a fórmula descrita por FALCONER \& MACKAY (1996).

$O$ coeficiente de relação médio (AR) de cada animal no pedigree foi também calculado. Esse coeficiente é definido como a probabilidade de um alelo qualquer, tomado ao acaso na população, pertencer a determinado indivíduo (GUTIÉRREZ \& GOYACHE, 2005). O AR pode ser interpretado como a representação do animal no pedigree completo, independentemente do conhecimento de seu próprio pedigree. $\mathrm{O}$ tamanho efetivo da população $\left(\mathrm{N}_{\mathrm{e}}\right)$ foi obtido seguindo a proposta de GUTIÉRREZ \& GOYACHE (2005), com base no cálculo de coeficientes de regressão do $\mathrm{F}$ individual sobre: i) o número de gerações completas traçadas, ii) o número máximo de gerações traçadas e iii) o número equivalente de gerações completas. $\mathrm{O}$ número efetivo de fundadores $\left(f_{e}\right)$ foi outro coeficiente utilizado para descrever a variabilidade genética da população. É definido como o número de fundadores com contribuições balanceadas que se poderia esperar para produzir a mesma diversidade genética como na população em estudo (LACY, 1989). O número efetivo de ancestrais $\left(f_{a}\right)$ complementa as informações fornecidas pelo $f_{e}$, contabilizando as perdas de variabilidade genética produzida pelo uso desbalanceado de indivíduos na reprodução. A soma 
das contribuições marginais de todos os ancestrais é igual a 1. Esses coeficientes $\left(f_{e}\right.$ e $\left.f_{a}\right)$ permitem levar em conta a perda de variabilidade genética produzida pelo efeito gargalo $\left(\mathrm{f}_{\mathrm{e}} / \mathrm{f}_{\mathrm{a}}\right)$. Foram calculados também o intervalo de gerações, dado pela média de idade dos pais ao nascimento de sua prole, mantida para reprodução, e a média de idade dos pais ao nascimento de sua prole, utilizada ou não para reprodução. Esses valores foram calculados para as quatro trajetórias de seleção (touros pais de touros, touros pais de vacas, vacas mães de touros e vacas mães de vacas), considerando-se apenas animais nascidos a partir de 1979 e cujas datas de nascimento foram conhecidas conjuntamente com a de seus pais. $\mathrm{O}$ arquivo de pedigree já recodificado foi utilizado para análise da estrutura da população, executada pelo programa ENDOG v. 4.8 (GUTIÉRREZ \& GOYACHE, 2005). Para o cálculo da média anual de endogamia, foram usados os coeficientes de endogamia dos animais com data de nascimento conhecida.

\section{RESULTADOS E DISCUSSÃO}

$\mathrm{Na}$ primeira geração de ancestrais da população, aproximadamente $38,9 \%$ dos animais tiveram ambos os pais desconhecidos, ou seja, a integridade do pedigree foi de $61 \%$. A porcentagem de avôs e bisavôs de origem paterna foi maior que a daqueles de origem materna, porém houve tendência de a quantidade de informação do pedigree dos ancestrais reduzir com o passar das gerações. $\mathrm{Na}$ segunda geração de ancestrais, a integridade foi de $32,65 \%$ e, nas gerações subsequentes, diminuiu progressivamente para 19,$41 ; 11,00 ; 5,13 ; 2,05 ; 0,69$; $0,18,0,04 ; 0,04$ e $0,04 \%$.

O nível de conteúdo de informação do pedigree (PCI) neste estudo, em número de ancestrais conhecidos (61\% na atual geração), e o PCI médio na população completa de 11 gerações $(0,12)$ foram inferiores aos relatados por STACHOWICZ et al. (2011) para a raça Holandesa no Canadá. Essas diferenças podem ser atribuídas às quantidades de informação utilizadas nos cálculos, ao número de gerações consideradas e ao tempo da fundação da raça. Tendo em vista a recente formação da raça Girolando (23 anos), os valores de PCI encontrados neste estudo podem ser considerados de qualidade moderada.

$\mathrm{O}$ arquivo de pedigree incluiu registros de 26.969 animais, tendo um $\mathrm{F}$ médio igual a $0,11 \%$ (Tabela 1). Do total de animais analisados, 818 $(3,03 \%)$ eram endogâmicos, com valor médio de $\mathrm{F}$ de $3,69 \%$. Dentre os animais endogâmicos, o F médio dos pais foi inferior ao das mães, porém superior ao das vacas (animais sem progênie). O número de animais endogâmicos foi maior na categoria das vacas (381), todavia houve maior proporção de animais endogâmicos na categoria pais $(6,1 \%)$. O F médio da população completa da raça Girolando foi menor que os relatados no Brasil por RIBEIRO et al. (2000), para a raça Santa Gertrudis $(3,95 \%)$, e REIS FILHO et al. (2010), para a raça Gir Leiteiro $(8,82 \%)$. Na Venezuela, TULLO et al. (2011) encontraram F médio de 3,62\% na raça Carora. $\mathrm{O}$ menor valor médio de $\mathrm{F}$ encontrado na população Girolando pode ser atribuído ao seu moderado PCI $(61 \%)$ e também ao patrimônio genético da raça, proporcionado pelos animais com distintas composições de Gir e Holandês que continuamente têm sido cadastrados em registro aberto pela $\mathrm{ABCG}$. Esses resultados indicam que o baixo valor do $\mathrm{F}$ médio $(0,11 \%)$ é devido ao baixo grau de parentesco entre os animais da raça Girolando no Brasil. O coeficiente de relação médio (AR) na população completa foi $0,13 \%$, ligeiramente maior nas vacas e nos pais e menor nas mães (Tabela 1). O valor médio do AR foi menor que os observados por REIS FILHO et al. (2010) na raça Gir Leiteiro $(2,1 \%)$ e por TULLO et al. (2011) na raça Carora $(5,88 \%)$. Esse menor valor de AR observado na população

Tabela 1 - Níveis de endogamia na população da raça Girolando.

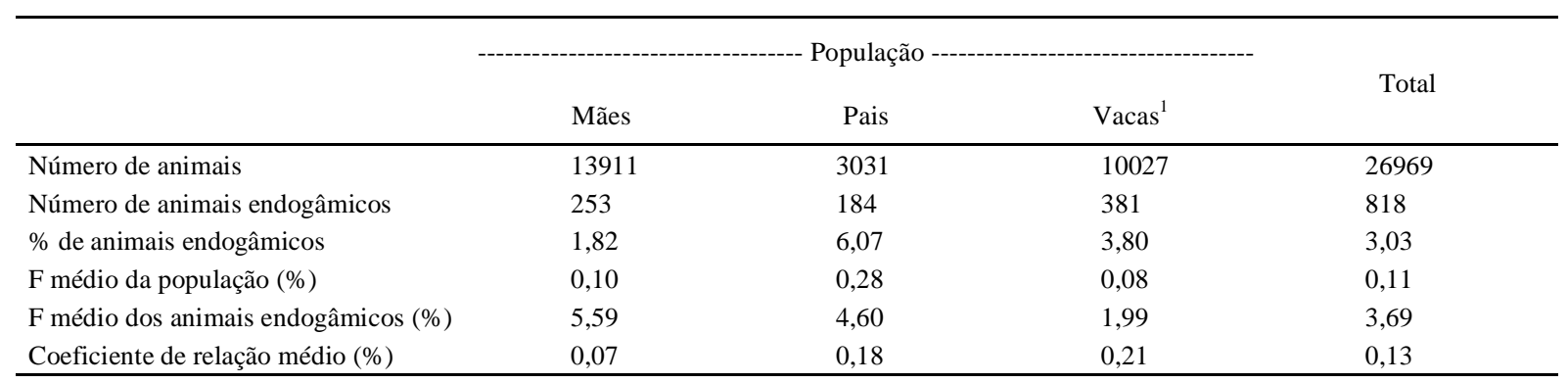

${ }^{1}$ animais sem progênie.

Ciência Rural, v.44, n.11, nov, 2014. 
Girolando pode ser atribuído ao fato de os criadores terem evitado o acasalamento de animais aparentados no período analisado.

As tendências dos valores médios de $\mathrm{F}$ em animais com data de nascimento (endogâmicos ou não) e em animais apenas endogâmicos foi similar ao longo dos anos, porém com magnitudes distintas (Figura 1). Os maiores níveis de endogamia foram atingidos no ano de 1993 , flutuando de $0,04 \%$ (em 1996) a $0,39 \%$ (em 1993) para todos os animais e de $0,78 \%$ (em 1996) a 14,58\% (em 1993) na população endogâmica. A partir de 1998, o coeficiente F dos animais endogâmicos tendeu à redução ao longo do tempo, numa taxa de $0,37 \%$ ao ano $\left(\mathrm{R}^{2}=70 \%\right)$. Isso pode ser explicado pelo fato de os animais recentemente registrados terem sido provenientes de acasalamentos de pais pouco aparentados, o que coincide com o início do teste de progênie de touros, em 1997, na raça Girolando. A introdução de grande número de novos animais, principalmente das raças Holandesa e Gir, contribuiu na redução da endogamia nos últimos anos.

O DF na população de animais Girolando apresentou tendência positiva ao longo do tempo avaliado, 1979 a 2008, comportamento que pode estar associado ao uso intensivo de reprodutores avaliados pelo teste de progênie da raça, à implementação de avaliação genética via BLUP, à adoção de inseminação artificial e à importação de sêmen de touros da raça Holandesa. No entanto, o aumento médio anual da endogamia foi de apenas $0,0075 \%\left(\mathrm{R}^{2}=0,81\right)$, bem abaixo dos níveis críticos sugeridos por NICHOLAS (1989), de 0,5\% por ano, como valores aceitáveis em programas de melhoramento. As médias de DF por tipo de geração considerada (Tabela 2) foram menores que o nível crítico recomendado pela FAO (FAO, 1998), que sugere evitar DF acima de 1\% por geração. Os baixos valores dos coeficientes $\mathrm{F}$ e $\mathrm{DF}$ indicam que as atuais práticas de acasalamento de animais nas fazendas, com fins de se evitar a endogamia, estão adequadas.

Os valores de $\mathrm{N}_{\mathrm{e}}$ foram 188,17; $1571,94 \mathrm{e}$ 369,33 nas gerações completas, gerações máximas e gerações equivalentes, respectivamente (Tabela 2). Segundo a FAO (1998), o nível crítico do $\mathrm{N}_{\mathrm{e}}$ é de 50 animais. Todavia, GODDARD \& SMITH (1990) sugeriram valores mínimos de 40 animais por geração para maximizar o ganho genético em uma população selecionada para produção de leite. $\mathrm{O}$ valor de $\mathrm{N}_{\mathrm{e}}$ $(188,17)$ encontrado para a população completa está acima desses níveis críticos e, ainda, acima dos níveis relatados para as raças Marchigiana e Bonsmara (SANTANA Jr. et al., 2012), Gir Leiteiro (REIS FILHO et al., 2010) e Brangus (STEYN et al., 2012).

$\mathrm{O} f$ observado na população foi de 551 e o aumento esperado da endogamia, devido à contribuição não-balanceada dos fundadores, foi de $0,04 \%$. Os $75 \%$ e $50 \%$ das contribuições genéticas para a população de referência da raça Girolando foram explicados por 2.726 e 467 ancestrais, respectivamente. $\mathrm{O} \mathrm{f}_{\mathrm{a}}$ foi 393 e a relação entre o

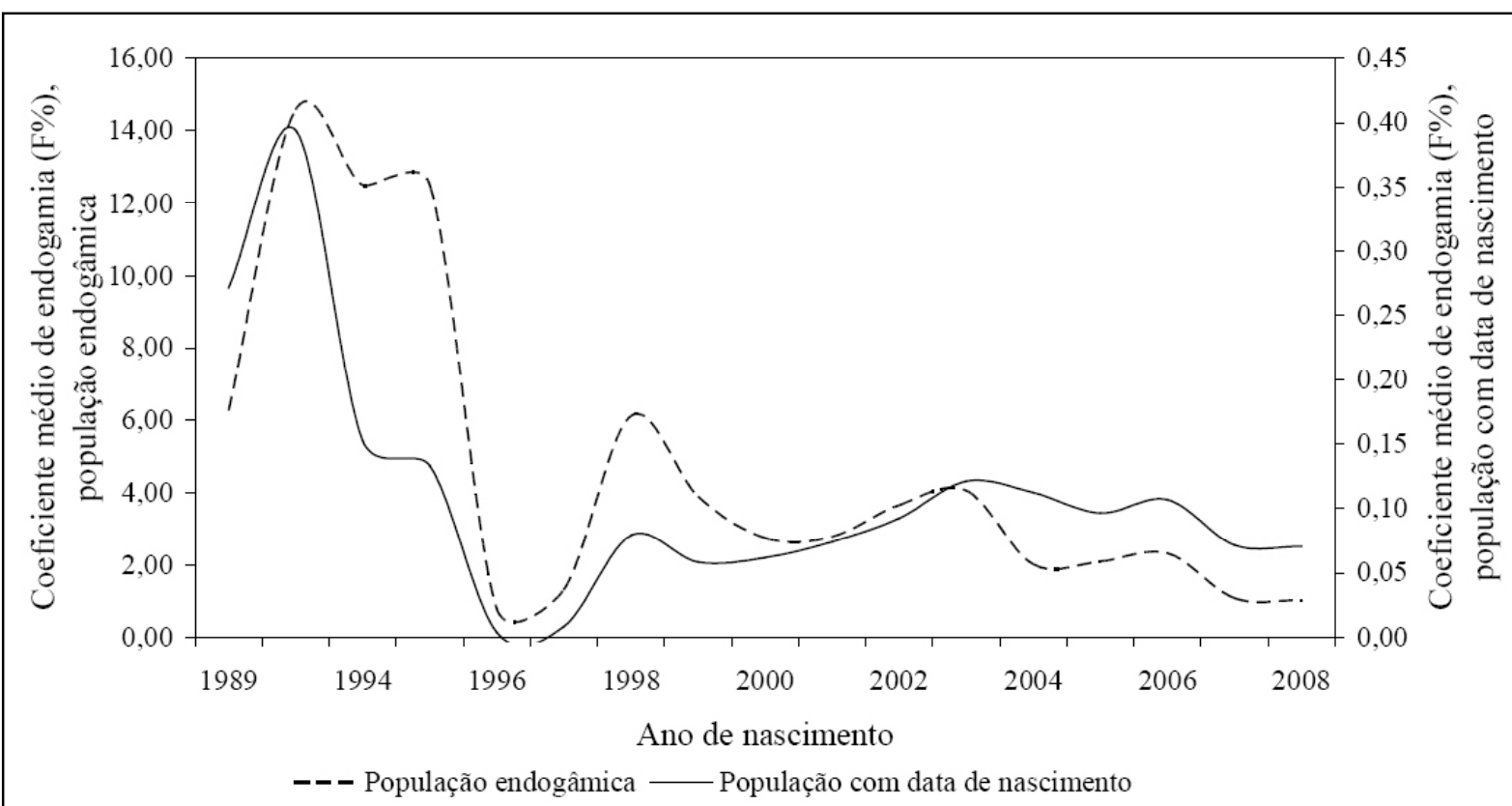

Figura 1 - Tendência da endogamia por ano de nascimento na população da raça Girolando com data de nascimento (n=12.439) e na população endogâmica ( $\mathrm{n}=440)$ durante o período de 1989 a 2008. 
Tabela 2 - Número médio e máximo de gerações traçadas, incremento de endogamia $(\Delta \mathrm{F})$ e tamanho efetivo da população $\left(\mathrm{N}_{\mathrm{e}}\right)$ por tipo de geração considerada.

\begin{tabular}{lcccc}
\hline Tipo de geração & $\mathrm{N}^{\mathrm{o}}$ médio & $\mathrm{N}^{\mathrm{o}}$ máximo & $\Delta \mathrm{F}$ & \multicolumn{1}{c}{$\mathrm{N}_{\mathrm{e}}$} \\
\hline Completas $^{1}$ & 0,80 & 4 & $0,27 \%$ & 188,17 \\
Máximas $^{2}$ & 2,87 & 11 & $0,03 \%$ & 1571,94 \\
Equivalentes $^{3}$ & 1,32 & 5,53 & $0,14 \%$ & 369,33 \\
\hline
\end{tabular}

${ }^{1}$ Geração mais distante em que todos os ancestrais são conhecidos. ${ }^{2}$ Número de gerações que separa o indivíduo de seu ancestral mais remoto. ${ }^{3}$ Somatório dos termos $(1 / 2)^{g}$ de todos ancestrais conhecidos, em que $g$ é o número de gerações que separa o indivíduo de cada ancestral conhecido.

número efetivo de fundadores e de ancestrais $\left(\mathrm{f}_{\mathrm{e}} / \mathrm{f}_{\mathrm{a}}\right)$ foi igual a 1,4, indicando que o efeito gargalo tem acontecido desde a formação da raça Girolando.

$O f_{e}$ e $f_{a}$ na população Girolando foram superiores aos relatados para algumas raças leiteira e de corte. No Brasil, REIS FILHO et al. (2010) relataram valores de 146 e 75 para $\mathrm{f}_{\mathrm{e}}$ e $\mathrm{f}_{\mathrm{a}}$, respectivamente, na raça Gir Leiteiro; enquanto SANTANA Jr. et al. (2012) obtiveram valores de 120 / 32 e 220 / 85 para $\mathrm{f}_{e} / \mathrm{f}_{\mathrm{a}}$, respectivamente, nas raças Marchigiana e Bonsmara. $\mathrm{Na}$ Venezuela, TULLO et al. (2011) encontraram valores de 40 e 30 para $f_{e}$ e $f_{a}$, respectivamente, na raça Carora. As principais causas dos altos valores de $f_{e}$ e $f_{a}$ na raça Girolando, em relação a essas raças, são as diferenças entre os tamanhos de população, os sistemas de produção e o procedimento de registro aberto adotado pela ABCG. O efeito gargalo observado neste estudo foi inferior aos relatados por REIS FIHO et al. (2010) para a raça Gir Leiteiro $(1,95)$ e por SANTANA Jr. et al. (2012), nas raças Marchigiana $(3,75)$ e Bonsmara (2,59); todavia, TULLO et al. (2011) encontraram valor semelhante na raça Carora (1,33). Essas diferenças podem ser devidas ao baixo número equivalente de gerações $(1,32)$ e ao uso desproporcional de alguns ancestrais.

A média do intervalo de geração de 5,26 anos é inferior aos de 8,41 anos para Gir Leiteiro (REIS FILHO et al., 2010) e 7,02 anos para Marchigiana (SANTANA Jr. et al., 2012); semelhante aos de 5,98 para Corora (TULLO et al., 2011) e 5,17 para Brangus (STEYN et al., 2012); e superior ao de 3,19 para Bonsmara (SANTANA Jr. et al., 2012). Os menores intervalos de geração estão associados às maiores taxas de reposição de reprodutores (touros e vacas), indicando que reprodutores jovens da raça Girolando têm sido utilizados.

A média do intervalo de geração foi maior nas trajetórias vacas mães de touros $(5,86)$ e touros pais de vacas $(5,60)$ em relação às trajetórias vacas mães de vacas $(4,97)$ e touros pais de touros $(5,24)$. Nos estudos de REIS FILHO et al. (2010) em Gir Leiteiro e de SANTANA Jr. et al. (2012) em Marchigiana, os intervalos de geração das trajetórias de seleção touros pais de touros e touros pais de vacas sempre foram superiores às trajetórias vacas mães de touros e vacas mães de vacas. Todavia, SANTANA Jr. et al. (2012) obtiveram na raça Bonsmara maiores intervalos de geração das trajetórias de seleção touros pais de touros e vacas mães de vacas. A ligeira superioridade do intervalo de geração médio nas vias gaméticas vacas mães de touros e touros pais de vacas é provavelmente atribuível ao fato de que determinados reprodutores têm sido utilizados por mais tempo que nas outras vias gaméticas para produzir progênie antes de realizar a seleção. Assim, o criador pode aumentar a intensidade seletiva e o progresso genético por geração, mas será inevitável o aumento no intervalo de gerações, com consequente redução no ganho genético por unidade de tempo.

\section{CONCLUSÃO}

Os valores do tamanho efetivo da população e do número efetivo de fundadores e ancestrais nos rebanhos Girolando encontram-se acima dos níveis críticos, indicando que as práticas de acasalamento para evitar a endogamia e manter a variabilidade na população foram adequadas durante o período avaliado. Ressalte-se, no entanto, a importância de se continuar com o monitoramento desses parâmetros na população a fim de prevenir futuros problemas de endogamia e perda de variabilidade.

\section{REFERÊNCIAS}

ASSOCIAÇÃO BRASILEIRA DE CRIADORES DE GIROLANDO (ABCG). Produção de leite no Brasil e participação da genética Girolando com ênfase em reprodução. Disponível em: <http://www.girolando.com.br/site/noticia. php?id=1859>. Acesso em: 12 out. 2011.

CORRALES, R. et al. Birth weight, reproduction traits and effects of inbreeding in Nicaraguan Reyna Creole cattle. Tropical Animal Health Production, v.43, p.1137-1143, 2011. Disponível em: <http://download.springer.com/static/pdf/853/art\%253A10.10 07\%252Fs11250-011-9814-4.pdf?auth66=1391007428_a5f9f230a af6f43a97d531c3f973faa3\&ext=.pdf $>$. Acesso em: 21 jan. 2014. doi: 10.1007/s11250-011-9814-4.

EAGLEN, S.A.E. et al. Evaluating alternate models to estimate genetic parameters of calving traits in UK Holstein-Friesian dairy cattle. Genetics Selection Evolution, v.44, n.1, p.23, 2012. Disponível em: <http://www.ncbi.nlm.nih.gov/pmc/articles/ PMC3468354/pdf/1297-9686-44-23.pdf >. Acesso em: 21 jan. 2014. doi: 10.1186/1297-9686-44-23. 
FALCONER, D.S.; MACKAY, T.F.C. Introduction to quantitative genetics. 4.ed. London: Longman Group, 1996. 464p.

FAO. Secondary guidelines for development of national farm animal genetic resources management plans: management of small populations at risk. Rome, Italy: Food and Agricultural Organization, 1988. 215p. Disponível em: <http://dad.fao.org/en/ refer/library/guidelin/sml-popn.pdf.>. Acesso em: 10 out. 2011.

GODDARD, M.G.; SMITH, C. Optimum number of bull sires in dairy cattle breeding. Journal of Dairy Science, v.73, p.1113-1122, 1990. Disponível em: <http://download.journals.elsevierhealth. com/pdfs/journals/0022.0302/PIIS0022030290787711.pdf >. Acesso em: 27 jul. 2013. doi: 10.3168/jds.S0022-0302(90)78771-1.

GUTIÉRREZ, J.P.; GOYACHE, F. A note on ENDOG: a computer program for analyzing pedigree information. Journal of Animal Breeding and Genetics, v.122, p.172-176, 2005. Disponível em: <http://onlinelibrary.wiley.com/doi/10.1111/ j.1439-0388.2005.00512.x/pdf>. Acesso em: 27 jul. 2013. doi: 10.1111/j.1439-0388.2005.00512.x

LACY, R.C. Analysis of founder representation in pedigrees: founder equivalents and founder genome equivalents. Zoo Biology, v.8, p.111-123, 1989. Disponível em: <http://onlinelibrary.wiley. com/doi/10.1002/zoo.1430080203/pdf >. Acesso em: 27 jul. 2013. doi: 10.1002/zoo.1430080203.

MACCLUER, J.W. et al. Inbreeding and pedigree structure in Standardbred horses. Journal of Heredity, v.74, p.394-399, 1983. Disponível em: <http://jhered.oxfordjournals.org/content/74/6/394>. Acesso em: 11 abr. 2014. doi: 10.1093/jhered/est079.

MEUWISSEN, T.H.E.; LUO, Z. Computing inbreeding coefficients in large populations. Genetics Selection Evolution, v.24, p.305-313, 1992. Disponível em: <http://www.gsejournal. org/content/pdf/1297-9686-24-4-305.pdf>. Acesso em: 27 jul. 2013. doi: 10.1186/1297-9686-24-4-305.

NICHOLAS, F.W. Incorporation of new reproductive technology in genetic improvement programmes. In: HILL, W.G.; MACKAY, T.F.C. (Eds.). Evolution and animal breeding. Wallingford, U.K: CABInternational, 1989. p.201-209.
REIS FILHO, J.C. et al. Population structure of Brazilian Gyr dairy cattle. Revista Brasileira de Zootecnia, v.39, p.2640-2645, 2010. Disponível em: <http://www.scielo.br/pdf/rbz/v39n12/ a12v39n12.pdf $>$. Acesso em: 27 jul. 2013. doi: 10.1590/S151635982010001200012

RIBEIRO, P.M.T. et al. Parâmetros genéticos e nível de endogamia em bovinos da raça Santa Gertrudis no Brasil. Arquivo Brasileiro de Medicina Veterinária e Zootecnia, v.52, n.6, p.641-646, 2000. Disponível em: <http://www.scielo.br/scielo. php?script=sci_arttext\&pid=S0102-09352000000600015\&lng =en\&nrm=iso>. Acesso em: 31 jan. 2014. doi: 10.1590/S010209352000000600015.

SANTANA Jr. M.L. et al. Pedigree analysis and inbreeding depression on growth traits in Brazilian Marchigiana and Bonsmara breeds. Journal of Animal Science, v.90, n.1, p.99108, 2012. Disponível em: <http://www.journalofanimalscience. org/content/90/1/99.full.pdf+html>. Acesso em: 21 jan. 2014. doi: 10.2527/jas.2011-4079.

SILVA, M.V.G.B. et al. Programa de melhoramento genético da raça Girolando - Teste de progênie: sumário de Touros 2010. Juiz de Fora: Embrapa Gado de Leite, 2010. 48p. (Embrapa Gado de Leite. Documentos, 131)

STEYN, J.W. et al. Preliminary report: Pedigree analysis of the Brangus cattle in South Africa. South African Journal of Animal Science, v.42, n.5, Suppl.1, p.511-514, 2012. Disponível em: <http://www.ajol.info/index.php/sajas/article/view/86371/76196>. Acesso em: 21 jan. 2014. doi: 10.4314\%2Fsajas.v42i5.14.

STACHOWICZ, K. et al. Rates of inbreeding and genetic diversity in Canadian Holstein and Jersey cattle. Journal of Dairy Science, v.94, p.5160-5175, 2011. Disponível em: <http:// download.journals.elsevierhealth.com/pdfs/journals/0022-0302/ PIIS0022030211005364.pdf>. Acesso em: 27 jul. 2013. doi: $10.3168 /$ jds.2010-3308.

TULLO, E. et al. Genetic variability and population structure in the Carora dairy breed. Italian Journal Animal Science, v.10, suppl.1, p.3, 2011. Disponível em: <http://www.aspajournal.it/index.php/ ijas/article/viewFile/ijas.2011.s1/2045>. Acesso em: 21 jan. 2014 